\title{
LA ECONOMÍA SOCIAL COMO ALTERNATIVA DE AUTOEMPLEO. EL PERFIL DEL EMPRENDEDOR/A EN LAS SOCIEDADES LABORALES ANDALUZAS
}

\author{
POR \\ Antonio Manuel CIRUELA-LORENZO ${ }^{1}$, \\ Mario CUADRADO SERRÁN ${ }^{2}$ y \\ Juan José PLAZA ANGULO 3
}

\section{RESUMEN}

El presente trabajo ofrece un análisis cuantitativo en relación al perfil del emprendedor/a que decide iniciar su actividad a través de una sociedad laboral en el contexto de la Comunidad Autónoma Andaluza. De esta forma, en primer lugar se presenta a la Economía Social como una alternativa muy importante de creación y fomento de empleo, que trata de aunar los objetivos económicos y sociales en el desarrollo de las actividades. Posteriormente, y centrándose en las sociedades laborales, se ofrecen datos actualizados sobre su importancia y repercusión para, posteriormente, y a través del análisis de dos muestras de sociedades laborales, establecer los atributos o características más significativas de éstos emprendedores/as.

Palabras Clave: Emprendimiento, Tercer Sector, Sociedad Laboral, Perfil Emprendedor, Andalucía

\footnotetext{
${ }^{1}$ Departamento Economía y Administración de Empresas de la Universidad de Málaga. Dirección de correo electrónico: $\underline{\text { acl@uma.es. }}$

2 Centro de Apoyo al Desarrollo Empresarial de Antequera (Málaga) de la Junta de Andalucía. Dirección de correo electrónico: mcuadrado@andaluciaemprende.es.

${ }^{3}$ Departamento Economía y Administración de Empresas de la Universidad de Málaga. Dirección de correo electrónico: juanjoseplaza@uma.es.

REVESCO N 122 - Tercer Cuatrimestre 2016 - ISSN: 1885-8031 - www.ucm.es/info/revesco

http://dx.doi.org/10.5209/rev_REVE.2016.v122.52019

Fecha de recepción: 01/05/2015

Fecha de aceptación: 21/12/2015
} 
Claves Econlit: P130, J540, L260

THE SOCIAL ECONOMY AS AN ALTERNATIVE OF SELF EMPLOYMENT. PROFILE OF THE ENTREPRENEUR IN THE ANDALUSIAN LABOUR COMPANIES

\begin{abstract}
This paper provides a quantitative and qualitative analysis of the profile of the entrepreneur who decides to start business through a labor society in the context of the Autonomous Community of Andalucia. Thus, first the Social Economy is presented as an important alternative to creating and promoting employment, which seeks to combine economic and social objectives in the development of activities. Subsequently, focusing on labor companies, updated its importance in the regional economy for later data and through the analysis of two samples are available, set the attributes or most significant characteristics of these entrepreneurs.
\end{abstract}

Keywords: Entrepreneurship, Social Economy, Workers cooperative, Entrepreneur Profile, Andalucía.

\title{
1. INTRODUCCIÓN: LAS SOCIEDADES LABORALES EN EL CONTEXTO DE LA ECONOMÍA SOCIAL
}

Este trabajo se centra en la Economía Social como un sector que pertenece a las denominadas como empresas de participación, que son aquellas en las que los socios actúan de manera activa en todos y cada uno de los procesos empresariales. Es decir, en los flujos reales, como proveedores de trabajo, en los flujos financieros aportando recursos a la estructura financiera y en los flujos informativo-decisionales tomando decisiones de forma democrática (Martín y Lejarriaga, 2011).

A este respecto, Pérez (2002) señala que las primeras empresas en adoptar la participación como seña de identidad fueron las de economía social y, por ello, deben ser consideradas empresas de participación. Habitualmente se ha considerado como tal a la 
cooperativa de trabajo asociado aunque también lo son las sociedades laborales (figura en la que se centra este trabajo), como ha entendido, entre otros autores, el profesor Lejarriaga (2002).

El Manifiesto-Programa deI I Congreso de la Economía Social celebrado en Madrid en 1993, organizado por el Comité Español Permanente de la Economía Social, define a la Economía Social como toda actividad económica, basada en la asociación de personas en entidades de tipo democrático y participativo, con la primacía de las aportaciones personales y de trabajo sobre el capital (CEPES, 1993). Por tanto, uno de los aspectos fundamentales por los que se caracterizan estas sociedades no es otro que la primacía otorgada al elemento humano en el desarrollo de su actividad, estableciéndose los objetivos y organización del trabajo en base a las necesidades y circunstancias de sus socios. Asi es, éstas empresas consideran al individuo como el centro de sus actividades, otorgándoles autonomía y fomentando una cultura basada en valores como la igualdad, la participación y la comunicación. Circunstancias por las que se puede establecer que, teóricamente, y en relación a estos aspectos, parten de una mejor posición para el desarrollo de sus actividades y la adaptación a los continuos cambios del entorno (Ciruela, 2006).

Pero, ¿qué es la Economía Social? La Ley 5/2011, de 29 de marzo, de Economía Social, la define como el conjunto de las actividades económicas y empresariales, que en el ámbito privado llevan a cabo aquellas entidades que, de conformidad con los principios recogidos en el artículo 4, persiguen bien el interés colectivo de sus integrantes, bien el interés general económico o social, o ambos (artículo 2). El referido artículo 4 establece los principios orientadores de esta forma:

a) Primacía de las personas y del fin social sobre el capital, que se concreta en gestión autónoma y transparente, democrática y participativa, que lleva a priorizar la toma de decisiones más en función de las personas y sus aportaciones de trabajo y servicios prestados a la entidad o en función del fin social, que en relación a sus aportaciones al capital social. 
b) Aplicación de los resultados obtenidos de la actividad económica principalmente en función del trabajo aportado y servicio o actividad realizada por las socias y socios o por sus miembros y, en su caso, al fin social objeto de la entidad.

c) Promoción de la solidaridad interna y con la sociedad que favorezca el compromiso con el desarrollo local, la igualdad de oportunidades entre hombres y mujeres, la cohesión social, la inserción de personas en riesgo de exclusión social, la generación de empleo estable y de calidad, la conciliación de la vida personal, familiar y laboral y la sostenibilidad.

d) Independencia respecto a los poderes públicos.

Por último, para referirse a entidades que se encuadran dentro de la Economía Social, el artículo 5 de ésta misma Ley señala que son las cooperativas, las mutualidades, las fundaciones y las asociaciones que lleven a cabo actividad económica, las sociedades laborales, las empresas de inserción, los centros especiales de empleo, las cofradías de pescadores, las sociedades agrarias de transformación y las entidades singulares creadas por normas específicas que se rijan por los principios orientadores.

En lo relativo a las sociedades laborales, según el artículo 1 de la Ley 44/2015, son aquellas sociedades anónimas o limitadas, en las que al menos la mayoría del capital social esté en manos de trabajadores que presten en ellas sus servicios retribuidos de manera personal y directa en virtud de contratos de trabajo indefinidos y sin que ninguno de ellos ostente más de una tercera parte de las acciones o participaciones en que se divida el capital social. Se requiere además que el número total de horas al año trabajadas por los trabajadores con contrato por tiempo indefinido que no sean socios, no exceda del $49 \%$ del total de las horas por año trabajadas por los socios trabajadores. Por otro lado, cabe la posibilidad de constituirla inicialmente con únicamente dos socios trabajadores con contrato indefinido, una sociedad laboral en la que los socios tengan más de la tercera parte del capital, en la que tanto el capital social como los derechos de voto estén distribuidos al $50 \%$ entre los dos socios constituyentes. Este caso deberán adaptarse a los términos generales en un plazo máximo de 36 meses.

REVESCO N 122 - Tercer Cuatrimestre 2016 - ISSN: 1885-8031 - www.ucm.es/info/revesco 
Cano (2002) se aproxima al concepto de sociedad laboral, no solo desde el ámbito legal sino también desde el ámbito económico, desde tres enfoques:

a) Perspectiva macroeconómica: instrumento de la política económica del Estado Social en orden a una eficaz intervención de los poderes públicos en el mercado de trabajo con el fin de luchar contra el desempleo. En este sentido, Alguacil (2007) considera que las sociedades laborales constituyen un instrumento eficaz en la creación y mantenimiento de puestos de trabajo estables.

b) Perspectiva teoría factores de producción: permite a los trabajadores no la venta de su fuerza de trabajo sino la puesta en común de la misma. De este modo el factor trabajo pasa a ocupar la posición preeminente y el factor capital una posición subordinada en orden a la fijación de la política empresarial y para la determinación del destino del excedente empresarial. .

c) Perspectiva teoría económica: instrumento legal alternativo y más eficiente, en determinados supuestos y para ciertos cometidos, que las formas convencionales de organización de la actividad empresarial. Con ella se procura el mantenimiento de unos puestos de trabajo, normalmente estables y de calidad, a través de la realización de una actividad empresarial con sometimiento a la racionalidad del mercado (Valpuesta y Barberena, 1998).

En definitiva, en las Sociedades Laborales el trabajo es el objeto esencial (López, 2006) y esta mayor integración de éste factor en los fines de la empresa y en el establecimiento de la política de la misma provoca un fuerte aumento de la productividad, mostrando los trabajadores niveles motivacionales superiores a las empresas no participadas (Melgarejo, Arcelus y Simón, 2007). Motivación que es aún mayor por el hecho de que han de ser capaces de conciliar los objetivos sociales de búsqueda de un empleo estable y de calidad por parte de los socios, con los objetivos puramente económicos (Martín, Lejarriaga e Iturrioz, 2007). 
Estos rasgos conceptuales, unidos a la pequeña dimensión y a que suelen constituirse con capital propio de los socios trabajadores, podría permitir la afirmación de que las Sociedades Laborales son, al fin y al cabo, empresas de trabajo asociado con una vestidura jurídica distinta a la de las cooperativas, mostrándose como un instrumento que hace posible la plena y consciente identificación entre el interés social y el interés económico.

\section{LA SOCIEDAD LABORAL ANDALUZA EN CIFRAS}

Si se analiza la distribución de sociedades laborales constituidas en Andalucía en el período 2003-2014, se puede observar que Málaga es la provincia donde se constituyen un mayor número de éstas con 1.524 (21,30\%), seguida de la provincia de Sevilla con 1.319 $(18,43 \%)$. Por otra parte, Granada $(9,69 \%)$ y Jaén $(8,86 \%)$ ocupan las últimas posiciones. Destacar como Málaga y Sevilla registran aproximadamente el 40\% del total (véase Tabla 1).

Tabla 1. Creación de Sociedades Laborales en Andalucía. 2003-2014

\begin{tabular}{|c|c|c|c|c|c|c|c|c|c|}
\hline AÑO & ALMERÍA & CÁDIZ & CÓRDOBA & GRANADA & HUELVA & JAÉN & MÁLAGA & SEVILLA & TOTAL \\
\hline $\mathbf{2 0 0 3}$ & 180 & 160 & 148 & 148 & 158 & 141 & 300 & 285 & $\mathbf{1 . 5 2 0}$ \\
\hline $\mathbf{2 0 0 4}$ & 161 & 135 & 135 & 130 & 109 & 128 & 273 & 246 & $\mathbf{1 . 3 1 7}$ \\
\hline $\mathbf{2 0 0 5}$ & 124 & 131 & 98 & 90 & 116 & 78 & 193 & 197 & $\mathbf{1 . 0 2 7}$ \\
\hline $\mathbf{2 0 0 6}$ & 66 & 55 & 75 & 71 & 69 & 42 & 134 & 128 & $\mathbf{6 4 0}$ \\
\hline $\mathbf{2 0 0 7}$ & 58 & 68 & 80 & 63 & 75 & 58 & 163 & 108 & $\mathbf{6 7 3}$ \\
\hline $\mathbf{2 0 0 8}$ & 39 & 38 & 49 & 43 & 44 & 32 & 103 & 68 & $\mathbf{4 1 6}$ \\
\hline $\mathbf{2 0 0 9}$ & 19 & 17 & 39 & 24 & 29 & 29 & 111 & 62 & $\mathbf{3 3 0}$ \\
\hline $\mathbf{2 0 1 0}$ & 28 & 28 & 46 & 34 & 56 & 29 & 64 & 46 & $\mathbf{3 3 1}$ \\
\hline $\mathbf{2 0 1 1}$ & 25 & 29 & 33 & 26 & 25 & 26 & 73 & 59 & $\mathbf{2 9 6}$ \\
\hline $\mathbf{2 0 1 2}$ & 21 & 21 & 36 & 25 & 27 & 28 & 49 & 40 & $\mathbf{2 4 7}$ \\
\hline $\mathbf{2 0 1 3}$ & 19 & 26 & 23 & 20 & 15 & 32 & 41 & 37 & $\mathbf{2 1 3}$ \\
\hline $\mathbf{2 0 1 4}$ & 13 & 7 & 10 & 19 & 22 & 11 & 20 & 43 & $\mathbf{1 4 5}$ \\
\hline TOTAL & $\mathbf{7 5 3}$ & $\mathbf{7 1 5}$ & $\mathbf{7 7 2}$ & $\mathbf{6 9 3}$ & $\mathbf{7 4 5}$ & $\mathbf{6 3 4}$ & $\mathbf{1 . 5 2 4}$ & $\mathbf{1 . 3 1 9}$ & $\mathbf{7 . 1 5 5}$ \\
\hline$\%$ & $10,52 \%$ & $9,99 \%$ & $10,79 \%$ & $9,69 \%$ & $10,41 \%$ & $8,86 \%$ & $21,30 \%$ & $18,43 \%$ & $100 \%$ \\
\hline
\end{tabular}

Fuente: Elaboración propia en base a Registro Administrativo de Sociedades Laborales de Andalucía (R@slA).

Tal y como se observa en la figura 2, la evolución de sociedades laborales creadas en Andalucía se caracteriza por una tendencia negativa, confirmando el impacto de la crisis actual. Dicha tendencia sólo se invierte con un ligero incremento en 2007 (11,6\%), 
continuando su tendencia descendente hasta la actualidad, lo cual es una de las razones por las que se ha decidido abordar a este tipo de sociedades en el presente trabajo.

Gráfico 1. Evolución número SL registradas en Andalucía Período 2003 - 2014.

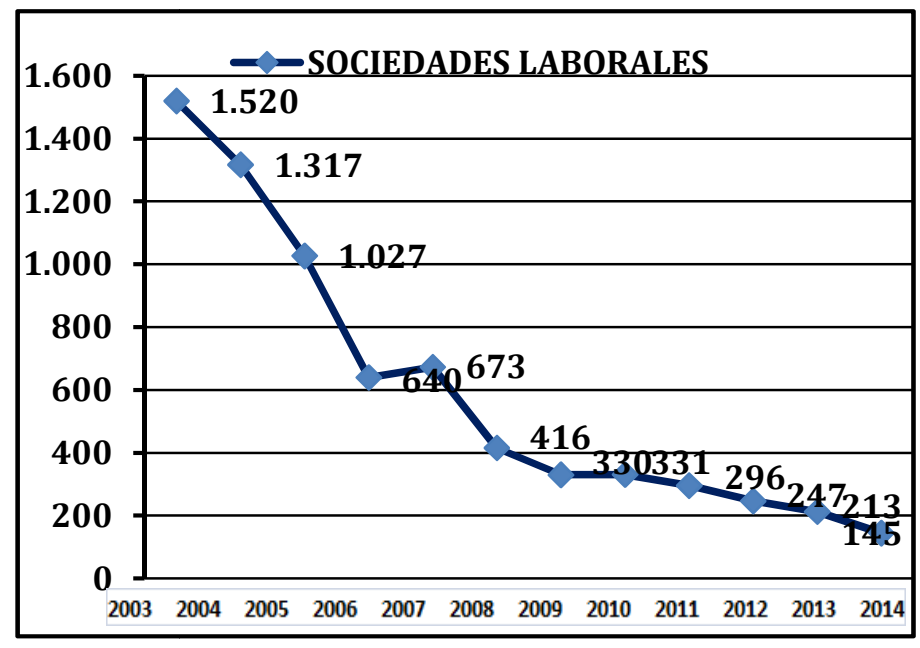

Fuente: Elaboración propia en base a Registro Administrativo de Sociedades Laborales de Andalucía (R@slA).

En lo que se refiere al Capital Social de nueva creación, éste asciende a 180,548.176 millones de euros en el periodo analizado (2003-2014). Este capital se distribuye según aportaciones de socios de clase laboral, (socios trabajadores que aportan capital y trabajo), por valor de 141,372.829 millones de euros, lo que representa el 78,30\%, y aportaciones económicas de socios de clase general (socios capitalistas que sólo aportan capital) por importe de 38,143.853 millones de euros $(21,13 \%)$, mientras que las aportaciones realizadas por personas jurídicas ascienden a 1,031.495 millones de euros, representando el 0,57\% del montante global (Registro Administrativo de Sociedades Laborales de Andalucía- R@ @lA).

El importe medio de capital asciende a $25.234 €$, siendo Sevilla la provincia con mayor capital suscrito $(41, .476 .830$ millones de euros - 22,97\%) y Cádiz la que registra una menor cifra $(14,658.852$ millones de euros - 8,12\%). 
Tabla 2. Socios de Sociedades Laborales en Andalucía según tipo y sexo

\begin{tabular}{|c|c|c|c|c|c|c|c|c|c|}
\hline TIPO & ALMERÍA & CÁDIZ & CÓRDOBA & GRANADA & HUELVA & JAÉN & MÁLAGA & SEVILLA & TOTAL \\
\hline Varones & 1.240 & 1.375 & 1.476 & 1.252 & 1.389 & 1.284 & 2.666 & 2.632 & $\mathbf{1 3 . 3 1 4}$ \\
\hline Mujeres & 565 & 560 & 585 & 512 & 508 & 401 & 1.209 & 1.073 & $\mathbf{5 . 4 1 3}$ \\
\hline $\begin{array}{c}\text { Socios } \\
\text { Trabajadores }\end{array}$ & $\mathbf{1 . 8 0 5}$ & $\mathbf{1 . 9 3 5}$ & $\mathbf{2 . 0 6 1}$ & $\mathbf{1 . 7 6 4}$ & $\mathbf{1 . 8 9 7}$ & $\mathbf{1 . 6 8 5}$ & $\mathbf{3 . 8 7 5}$ & $\mathbf{3 . 7 0 5}$ & $\mathbf{1 8 . 7 2 7}$ \\
\hline \% & $\mathbf{9 , 6 4 \%}$ & $\mathbf{1 0 , 3 3 \%}$ & $\mathbf{1 1 , 0 1 \%}$ & $\mathbf{9 , 4 2 \%}$ & $\mathbf{1 0 , 1 3 \%}$ & $\mathbf{9 , 0 0 \%}$ & $\mathbf{2 0 , 6 9 \%}$ & $\mathbf{1 9 , 7 8 \%}$ & $\mathbf{1 0 0 \%}$ \\
\hline Varones & 299 & 271 & 320 & 312 & 274 & 323 & 640 & 527 & $\mathbf{2 . 9 6 6}$ \\
\hline Mujeres & 446 & 387 & 489 & 441 & 476 & 421 & 866 & 706 & $\mathbf{4 . 2 3 2}$ \\
\hline $\begin{array}{c}\text { Socios } \\
\text { Capitalistas }\end{array}$ & $\mathbf{7 4 5}$ & $\mathbf{6 5 8}$ & $\mathbf{8 0 9}$ & $\mathbf{7 5 3}$ & $\mathbf{7 5 0}$ & $\mathbf{7 4 4}$ & $\mathbf{1 . 5 0 6}$ & $\mathbf{1 . 2 3 3}$ & $\mathbf{7 . 1 9 8}$ \\
\hline $\boldsymbol{\%}$ & $\mathbf{1 0 , 3 5}$ & $\mathbf{9 , 1 4}$ & $\mathbf{1 1 , 2 4}$ & $\mathbf{1 0 , 4 6}$ & $\mathbf{1 0 , 4 2}$ & $\mathbf{1 0 , 3 4}$ & $\mathbf{2 0 , 9 2}$ & $\mathbf{1 7 , 1 3}$ & $\mathbf{1 0 0}$ \\
\hline Varones & 1.539 & 1.646 & 1.796 & 1.564 & 1.663 & 1.607 & 3.306 & 3.159 & $\mathbf{1 6 . 2 8 0}$ \\
\hline Mujeres & 1.011 & 947 & 1.074 & 953 & 984 & 822 & 2.075 & 1.779 & $\mathbf{9 . 6 4 5}$ \\
\hline TOTAL & $\mathbf{2 . 5 5 0}$ & $\mathbf{2 . 5 9 3}$ & $\mathbf{2 . 8 7 0}$ & $\mathbf{2 . 5 1 7}$ & $\mathbf{2 . 6 4 7}$ & $\mathbf{2 . 4 2 9}$ & $\mathbf{5 . 3 8 1}$ & $\mathbf{4 . 9 3 8}$ & $\mathbf{2 5 . 9 2 5}$ \\
\hline \% & $\mathbf{9 , 8 4}$ & $\mathbf{1 0 , 0 0}$ & $\mathbf{1 1 , 0 7}$ & $\mathbf{9 , 7 1}$ & $\mathbf{1 0 , 2 1}$ & $\mathbf{9 , 3 7}$ & $\mathbf{2 0 , 7 6}$ & $\mathbf{1 9 , 0 5}$ & $\mathbf{1 0 0}$ \\
\hline
\end{tabular}

Fuente: Elaboración propia en base a Registro Administrativo de Sociedades Laborales de Andalucía (R@slA).

En lo que respecta al número de socios trabajadores, estas empresas generaron un total de 18.727 nuevos socios trabajadores, de los cuales un $71,10 \%$ eran hombres frente a un $28,90 \%$ de mujeres.

Más concretamente, las provincias de Andalucía con mayor número de socios trabajadores son Málaga con 3.875 (20,69\%) y Sevilla con 3.705 (19,78\%). Jaén, por el contrario, con 1.685 socios trabajadores $(9 \%)$ es la provincia que presenta un menor número. Por otra parte, los socios con participaciones sociales de clase general (socios capitalistas) ascienden a 7.198 , de los cuales el $41,21 \%$ son varones y el $58,79 \%$ mujeres. De esta forma, la provincia con mayor número de socios de clase general vuelve a ser Málaga (1.506 socios 20,92\%), seguida de la provincia de Sevilla (1.233 - 17,13\%). Cádiz con 658 socios capitalistas $(9,14 \%)$ es la provincia andaluza con menor número de socios de clase general. 
Si se analiza la evolución de los socios trabajadores, se puede apreciar una tendencia negativa con grandes oscilaciones periódicas interanuales, experimentando un leve incremento en el ejercicio 2007 (4,36\%). Tendencia, lógicamente, acorde con la evolución del número de sociedades laborales creadas en dicho periodo.

Gráfico 2. Evolución número de socios trabajadores iniciales registrados en Sociedades Laborales de Andalucía

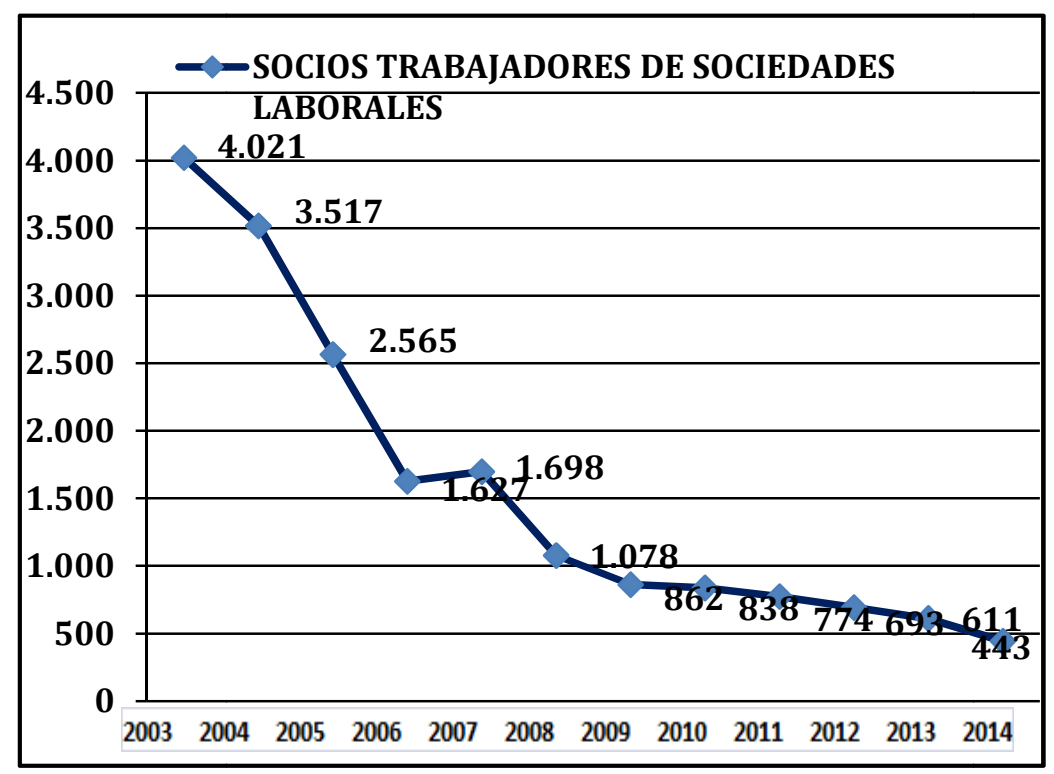

Fuente: Elaboración propia en base a Registro Administrativo de Sociedades Laborales de Andalucía (R@slA)

\section{LA FIGURA DEL EMPRENDEDOR EN LAS EMPRESAS DE ECONOMÍA SOCIAL}

Sin lugar a dudas una de las características fundamentales de la Economía Social, y más concretamente de las sociedades laborales es la posibilidad de crear empleo por cuenta propia, de emprender. Shapero y Sokol (1982) establecen que la acción de emprender es el hecho de tomar la iniciativa, consolidar los recursos, gestionar la empresa, actuar con autonomía y asumir el riesgo. En esta misma línea, Stevenson, Roberts y Grousbeck (1985), consideran emprendimiento como un continuo de actuación en el que en un extremo se identifica un promotor que considera que puede "hacer pasar las cosas" y en el otro extremo un administrador eficiente de recursos que los combina para aprovechar las oportunidades. 
Para Wennekers y Thurik (1999) emprender es poner en marcha un nuevo proyecto empresarial asumiendo el riesgo que éste conlleva, a cambio de obtener un beneficio empresarial. Se trata de un motor de innovación, competitividad y crecimiento. Sin embargo hay un acuerdo generalizado en que involucra la toma de decisiones y, en particular, implica contar con las competencias necesarias para poder tomar dichas decisiones de la manera correcta, y esto abarca diversos aspectos o elementos a considerar. Más concretamente VanderWerf y Brush (1989) afirman que emprender es una actividad de negocios que consiste en una intersección de las siguientes conductas:

Creación: establecimiento de una nueva unidad de negocio.

Administración general: dirección apropiada para una buena utilización de recursos.

Innovación: generación y explotación comercial de nuevos productos, servicios procesos, mercados, sistemas de organización, etc.

Aceptación del riesgo: la capacidad para manejar el riesgo de fallas potenciales al tomar decisiones o realizar acciones.

Mejor desempeño: el intento por lograr altos niveles de desempeño o de crecimiento.

John Burch (1986), por su parte, agrega a la lista anterior más rasgos característicos de las personas emprendedoras:

Trabajadores incansables: trabajo-adictos (workaholics) que se enfocan a sus metas y trabajan incansablemente para alcanzarlas.

Optimistas: consideran que cualquier cosa es posible y que el momento es inmejorable para alcanzar metas y logros.

Orientación a la excelencia: su deseo de logro los lleva a hacer las cosas al mejor nivel posible para sentirse aun más orgullosos y satisfechos de lo alcanzado.

\subsection{Perfil del emprendedor en las sociedades laborales de Andalucía}

Un primer condicionante de los emprendedores en sociedades laborales es que en ellos recae el doble rol de trabajador y propietario o socio de la empresa. Esta premisa les dota de 
ciertas particularidades que se manifiestan en las demás características de estos emprendedores y de estas empresas (Cano, 2002).

Se trata, además, de un emprendedor colectivo, pues el mínimo de socios trabajadores indefinidos para poder constituir una sociedad laboral según la Ley 44/2015 es de tres, o de dos según la excepción prevista en el artículo 1.2. b). Por ello se considera que la figura del emprendedor, objeto del presente estudio, es el fundador, aquel que da vida a la empresa (Suárez, 1992), que va a fijar los objetivos, a establecer la estrategia empresarial y a tomar las decisiones oportunas para su consecución (Castillo et al., 2003).

En primer lugar, se va a centrar el análisis en la motivación por la que un individuo decide crear una sociedad laboral. La literatura sobre emprendimiento habla de dos grandes motivadores; de un lado, el afán por la independencia y la autorrealización (Díez, et al., 1995; Coduras, 2006) y, de otro, las perturbaciones de corte negativo que impulsan a las personas a tomar la iniciativa con el objetivo de buscar cierta estabilidad laboral (Coduras, 2006; Hisdrich, et. al., 2005). A nivel más concreto, sin lugar a dudas, la posibilidad de capitalización del desempleo (Martín, Lejarriaga e Iturrioz, 2005a, 2005b) es un factor que ha alimentado ambos motivadores

García, et. al. (2007) apuntan que la empresa es el reflejo de las personas que la impulsan y, por ello, es importante conocer los atributos personales del emprendedor, lo que también señala González (2006). Tras la revisión de la literatura y de acuerdo con el propósito del presente trabajo, se han identificado los siguientes atributos o características a considerar: responsabilidad y perseverancia (Caprara et. al., 1998; Barba y Martínez, 2006), conocimiento de sus limitaciones (Barba y Martínez, 2006; Rajadel, 2003), tolerancia hacia la ambigüedad y la incertidumbre (González, 2006), ambición y liderazgo (Moriano, 2005; González, 2006), asunción de riesgos calculados (Morris y Kuratko, 2002), autoconfianza y optimismo (Moriano, 2005), organización y administración de recursos (Rajadel, 2003; Olamendi, 1998), comunicación eficaz (Barba y Martínez, 2006), perspicacia y proactividad (González, 2006; Morris, et. al., 2002) formación y conocimientos adecuados (García y Wandosell, 2004; 
Hisrich, et. al., 2005; Martín, Hernangómez y Rodríguez, 2005) e innovación y creatividad (Moriano, 2005; Amabile, 1997).

En cuanto al proceso de creación de la nueva empresa, parece importante el hecho de la planificación de la actividad y la fijación de objetivos y estrategias (Aguirre y Santos, 2004), pero no únicamente desde el prisma de la elaboración de un plan de empresa sino de la conciencia real de los socios fundadores de la fijación de dichas pautas y del cumplimiento de las mismas, incidiendo especialmente en la forma de la toma de decisiones y en la delimitación y conocimiento de las responsabilidades de cada socio trabajador. Además, también se han de considerar la formación y los conocimientos de los socios trabajadores sobre la actividad empresarial que desarrollan (Sanz de Galdeano, 2008), lo que posibilitará el conocimiento de si se trata de emprendedores con experiencia previa en el sector en el que emprenden y, además, ayudará a identificar la causa de la motiviación para emprender.

Por su parte, los factores del entorno, que son aquellos que el propio emprendedor no puede controlar, se presentan como las principales barreras a la hora de la creación de la empresa y de su supervivencia futura. Por otra parte, se encuentran la situación competitiva del sector y la etapa del ciclo económico, como factores clave del entorno (García y Wandosell, 2004).

Pero, además, para el ámbito del estudio, las sociedades laborales andaluzas, se han identificado también las siguientes variables de análisis: el tamaño inicial y actual de la empresa (Blázquez, et. al., 2006, Pinillos, 2007), la burocracia y los procedimientos administrativos, la fiscalidad y los impuestos (Informe Consejo Económico y Social, 2005; Informe GEM Andalucía, 2013), la financiación (Peña , 2008; Melgarejo, et. al., 2007; Villalba, et. al., 2007), la capitalización o pago único de la prestación por desempleo como financiación propia en las sociedades laborales (Plaza y Álvarez. 2008; Coll y Cuñat, 2006), los factores culturales del entorno (Veciana, 2005), la innovación y transferencia de I+D (Segarra y Teruel, 2007; Veciana, 2005), el acceso a infraestructuras comerciales y profesionales (Informe GEM Andalucía, 2006) y la apertura al mercado (Castillo et. al., 2006), algunos de los cuales son analizados en el estudio empírico posterior.

REVESCO No 122 - Tercer Cuatrimestre 2016 - ISSN: 1885-8031 - www.ucm.es/info/revesco 
Una vez establecida la importancia de las sociedades laborales en el contexto andaluz, así como las bases teóricas de la figura del emprendedor, se va a llevar a cabo un análisis empírico que permitirá un acercamiento al perfil real del emprendedor en este tipo de sociedades. Para ello se hace referencia a dos estudios, uno basado en variables más numéricas, cuyos proceden del Registro Administrativo de Sociedades Laborales de Andalucía (R@slA) y otro que, usando fuentes primarias, ha permitido un acercamiento a la faceta más personal y decisional de dichos emprendedores. En la Tabla 3 se resumen las dos fichas técnicas.

Tabla 3. Fichas técnicas del análisis empírico

\begin{tabular}{|c|c|c|}
\hline Universo & \multicolumn{2}{|c|}{2979 sociedades laborales con domicilio social en Andalucía } \\
\hline Ámbito geográfico & \multicolumn{2}{|c|}{ Andalucía } \\
\hline Tamaño de la Muestra & $\begin{array}{c}1272 \text { Sociedades Laborales adscritas } \\
\text { al Programa Red Territorial de } \\
\text { Apoyo a Emprendedores }\end{array}$ & 340 Sociedades Laborales \\
\hline Metodología & $\begin{array}{c}\text { Recepción de inscripción en el } \\
\text { Registro y grabación por los Centros } \\
\text { de Apoyo } \\
\text { al Desarrollo Empresarial (CADE) }\end{array}$ & Muestreo Aleatorio simple \\
\hline Herramienta & $\begin{array}{c}\text { Aplic@: Plataforma Tecnológica } \\
\text { Andalucía Emprende }\end{array}$ & Encuesta telefónica \\
\hline Error Muestral & $+/-2,08 \%$ & $+/-5 \%$ \\
\hline Nivel de confianza & \multicolumn{2}{|c|}{$95 \% \mathrm{Z}=1,96 ;$ Alfa $=0,05 ; \mathrm{P}=\mathrm{Q}=50 \%$} \\
\hline Período recogida datos & 01/01/2003 a $31 / 12 / 2014$ & 01/10/2014 a 30/11/2014 \\
\hline
\end{tabular}

Fuente: Elaboración propia.

\subsection{Análisis de Resultados}

Tras el análisis de la información obtenida se han obtenido los siguientes resultados: 
Género: los socios varones son mayoría con el $68,60 \%$, mientras que las socias representan el 31,40\%. Esta distribución se acentúa aún más en el caso de las Sociedades Anónimas Laborales (SAL) donde los emprendedores de género masculino representan el $89,80 \%$ en el período de referencia analizado.

Edad: la edad de mayor frecuencia es de 30 años, siendo la media de 35,36 años, con una desviación típica de 10,79, lo que implica, en términos generales, una edad promedio inferior a la media del conjunto de emprendedores españoles (38,9 años según Congregado et al., 2008).

Gráfico 3. Distribución de emprendedores de Sociedades Laborales según forma jurídica por edad

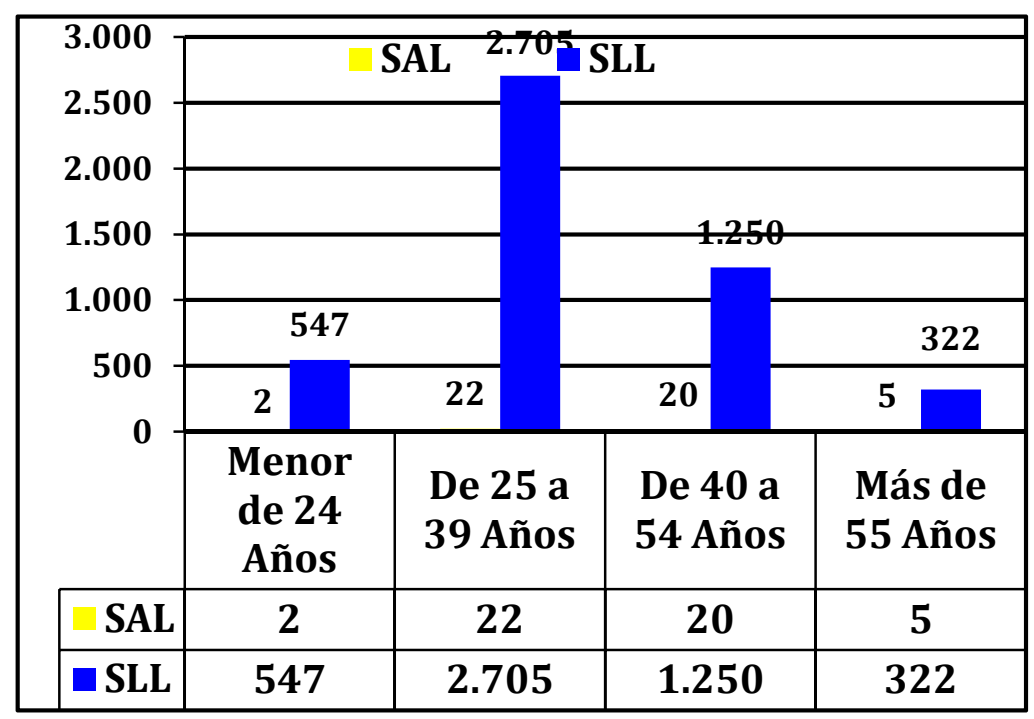

Fuente: Elaboración propia a partir de la muestra obtenida en los CADE

Formación: el 25,30\% de los emprendedores analizados ha cursado estudios superiores en la Universidad, a diferencia del 11,68\% que manifiesta no haber realizado estudios. Un 30,62\% ha realizado la Enseñanza Secundaria Obligatoria (ESO o equivalente), el 17,36\% tiene estudios de Formación Profesional (FP) y un 14,11\% ha realizado estudios de BUP-COU. Asimismo, el grupo mayoritario según tipo de sociedad y formación realizada, corresponde al grupo de emprendedores de Sociedades Limitadas Laborales (SLL) con un grado formativo alcanzado igual al de Enseñanza Secundaria Obligatoria (ESO) o equivalente $(30,31 \%)$.

REVESCO No 122 - Tercer Cuatrimestre 2016 - ISSN: 1885-8031 - www.ucm.es/info/revesco 
Tabla 4. Distribución de emprendedores de empresas de Economía Social de nueva creación en el período 2003-2012 según nivel formativo

\begin{tabular}{|c|c|c|c|c|c|c|c|c|}
\hline & Sin Estudios & ESO & FP & $\begin{array}{c}\text { BUP } \\
\text { COU }\end{array}$ & Diplomado & Ingeniería & Licenciado & TOTAL \\
\hline SAL & 9 & 15 & 9 & 8 & 3 & 0 & 5 & 49 \\
\hline SLL & 560 & 1.477 & 837 & 725 & 528 & 139 & 558 & $\mathbf{4 . 8 2 4}$ \\
\hline TOT. & $\mathbf{5 6 9}$ & $\mathbf{1 . 4 9 2}$ & $\mathbf{8 4 6}$ & $\mathbf{7 3 3}$ & $\mathbf{5 3 1}$ & $\mathbf{1 3 9}$ & $\mathbf{5 6 3}$ & $\mathbf{4 . 8 7 3}$ \\
\hline$\%$ & $\mathbf{1 1 , 6 8}$ & $\mathbf{3 0 , 6 2}$ & $\mathbf{1 7 , 3 6}$ & $\mathbf{1 5 , 0 4}$ & $\mathbf{1 0 , 9 0}$ & $\mathbf{2 , 8 5}$ & $\mathbf{1 1 , 5 5}$ & $\mathbf{1 0 0}$ \\
\hline
\end{tabular}

Fuente: Elaboración propia a partir de la muestra obtenida en los CADE.

Actividad económica: el sector económico más representativo de la muestra objetivo de análisis es el sector terciario o servicios con un total de 664 sociedades laborales $(52,20 \%)$, seguido muy de cerca por el sector industria con $598(47,01 \%)$ y, en último lugar a mucha distancia, el sector primario con sólo 10 sociedades (0,79\%). Esta distribución sectorial viene a reflejar la progresiva mutación de la actividad productiva/económica acaecida en las últimas décadas en la economía regional andaluza, caracterizada por un significativo cambio en el modelo productivo motivado por el continuo trasvase desde los sectores económicos tradicionales primario y secundario hacia el sector terciario o servicios, en paralelo al continuo proceso de terciarización de la economía nacional española.

Gráfico 4.Distribución de actividades económicas de Sociedades Laborales en el período 2003-2014

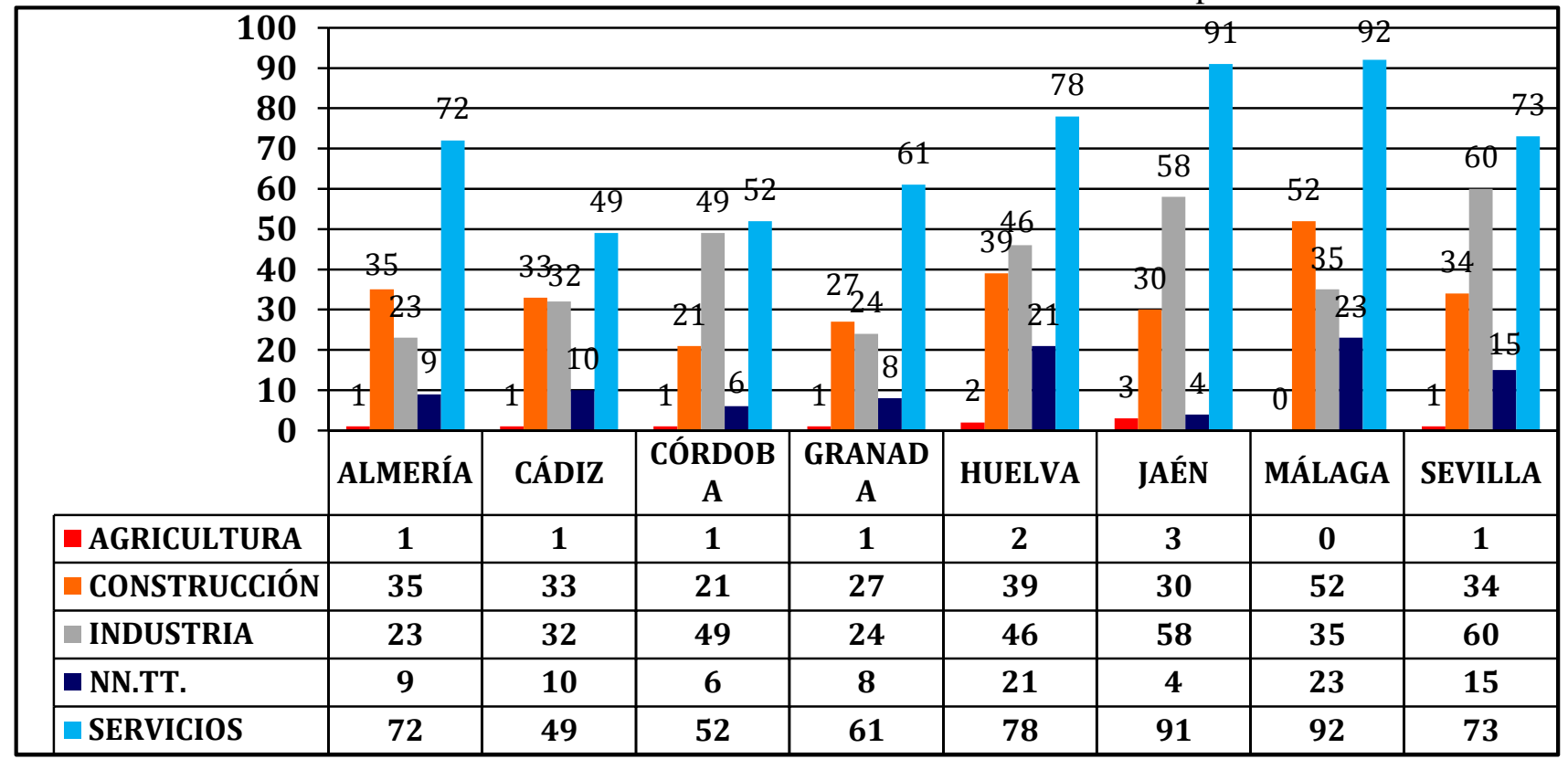

Fuente: Elaboración propia. Datos a partir de la muestra obtenida en los CADE. 
Por provincias, Málaga es la que mayor número de Sociedades Laborales posee en el sector servicios con un total de $92(16,20 \%)$ y en el de Construcción (52), mientras que Sevilla es la provincia que más Sociedades Laborales posee en industria (60) y la tercera en nuevas tecnologías con $15(15,62 \%)$. Por otra parte, Jaén es la provincia más representativa en el sector de Agricultura con 3 sociedades (30\%).

Después de realizar este análisis cuantitativo, se propone abordar de una forma más directa el perfil del emprendedor, tratando de obtener variables más relacionadas con su forma de pensar y actuar en el proceso de emprendimiento. De esta forma, los principales resultados obtenidos tras el análisis de los datos de la encuesta se exponen a continuación:

\section{a) Motivación para emprender}

La opción principal por la que los socios de una sociedad laboral se convierten en emprendedores se reparte de manera similar entre dos variables. De este modo, el 45,22\% afirma que se convirtió en empresario por la necesidad de conseguir un empleo estable y unas condiciones laborales que no le ofrecía el mercado de trabajo (motivación negativa), frente a un 54,78\% que emprendió por la satisfacción personal que supone crear tu propio medio de vida (motivación positiva).

Al considerar el sector de actividad, el comportamiento motivacional es similar, tal y como puede observarse en la siguiente tabla. Si bien, el sector de la construcción es el que cuenta con una mayor motivación positiva, lo cual puede deberse a la oportunidad que brindó la etapa de crecimiento inmobiliario de años anteriores. Por su parte, el sector servicios es el que cuenta con un mayor porcentaje de emprendedores que inician una sociedad laboral como consecuencia de motivaciones de corte negativo. 
Tabla 5. Motivación para emprender por sector de actividad

\begin{tabular}{|l|c|c|c|c|}
\hline & Agricultura & Industria & Construcción & Servicios \\
\hline Empleo estable y de calidad & $42,86 \%$ & $41,67 \%$ & $37,04 \%$ & $48,28 \%$ \\
\hline Independencia laboral & $57,14 \%$ & $58,33 \%$ & 62,965 & $51,72 \%$ \\
\hline
\end{tabular}

Fuente: Elaboración propia.

\section{b) Principales atributos personales}

El $39,05 \%$ de los emprendedores en sociedades laborales se manifiestan como responsables y perseverantes. En segundo lugar, se consideran optimistas y con suficiente autoconfianza en su actividad y sus posibilidades (31,27\%). Por su parte, el $10,66 \%$ se considera con capacidad de liderazgo, encontrando cifras similares en cuanto a la intuición o perspicacia y la proactividad (10,37\%). Por último, se comprueba como tan sólo un 8,65\% de los emprendedores en sociedades laborales tolera cierta ambigüedad y riesgo.

Gráfico 5. Principales atributos del emprendedor

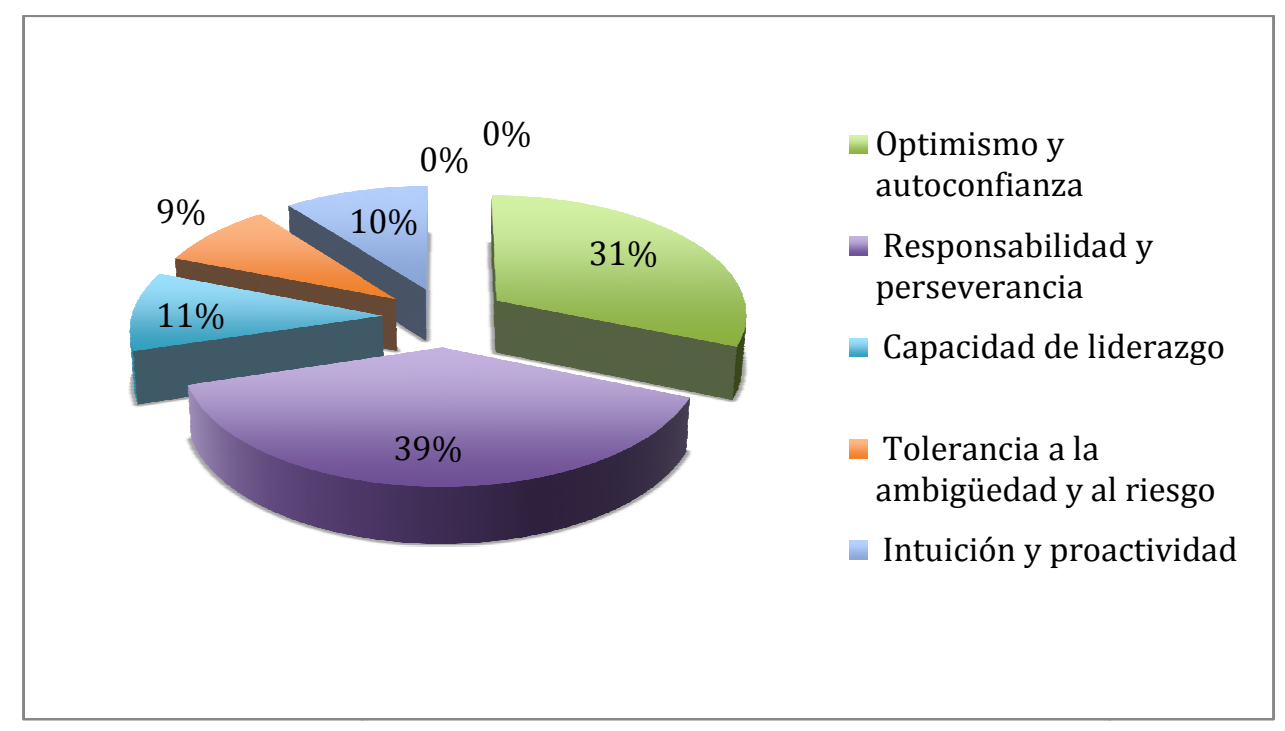

Fuente: Elaboración propia.

\section{c) Formación y capacitación profesional}

En cuanto a la formación es importante señalar que no únicamente se ha de tener en cuenta la formación profesional sino también aquélla fruto de la experiencia laboral previa. 
En relación a los resultados obtenidos, casi la totalidad de los socios trabajadores de las sociedades laborales considera que cuenta con la formación profesional, experiencia y conocimientos idóneos para desarrollar su actividad.

Gráfico 6. La formación profesional, experiencia y conocimientos del sector son los adecuados

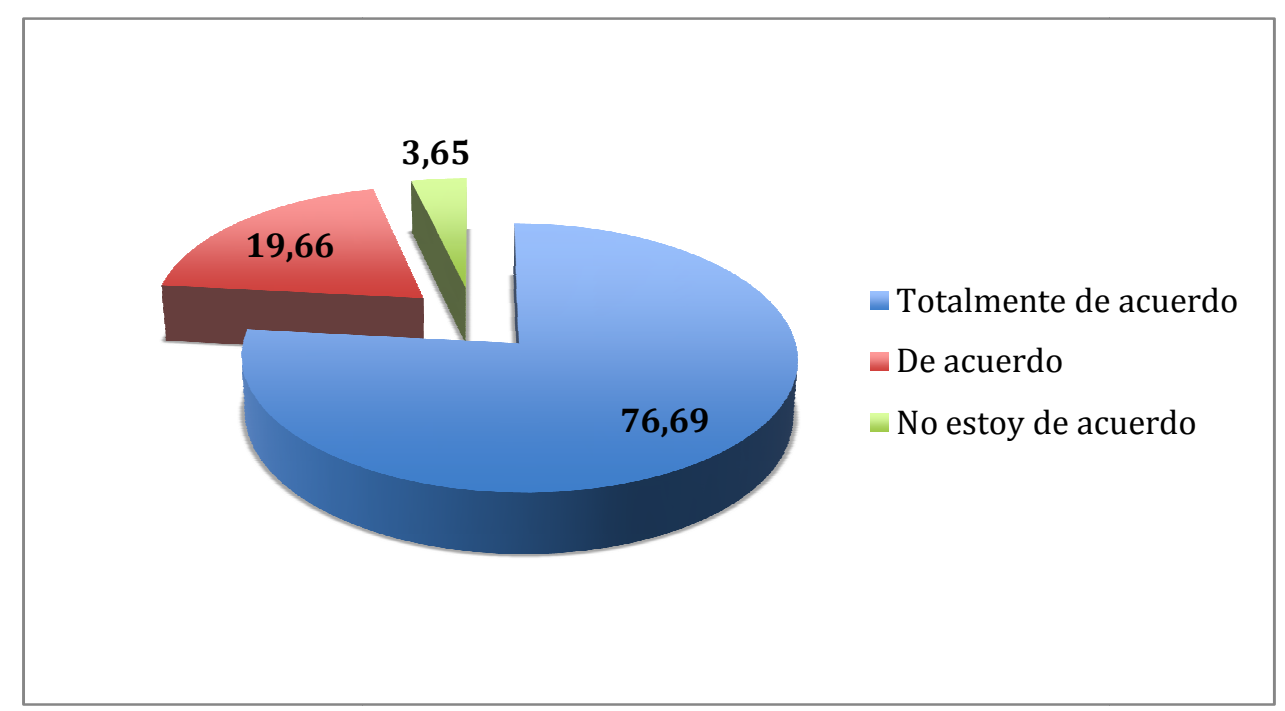

Fuente: Elaboración propia.

Estos datos pueden ser reflejo del hecho de que las sociedades laborales suelen constituirse en multitud de ocasiones por profesionales que han perdido su puesto de trabajo habitual y que mediante la constitución de este tipo de sociedades retoman su actividad profesional habitual, por lo que su pérfil se corresponde al de un trabajador cualificado.

\section{d) Gestión empresarial}

En primer lugar, se identifican las empresas que han marcado estrategias desde su nacimiento, con la consecuente planificación de la actividad y el reparto de funciones y responsabilidades de cada uno de los socios, bajo el objetivo de lograr el mayor control posible de todos los aspectos relacionados con su actividad. De esta forma, el 72,75\% de estas empresas afirma que desde el primer momento se planificó la actividad productiva de la empresa y se definieron las funciones y responsabilidades que cada uno de los socios 
desempeñaría dentro de la misma. Únicamente un 4,78\% de las sociedades laborales entiende que no lo hace.

Gráfico 7. Sociedades laborales que planificaron su actividad y definieron las responsabilidades

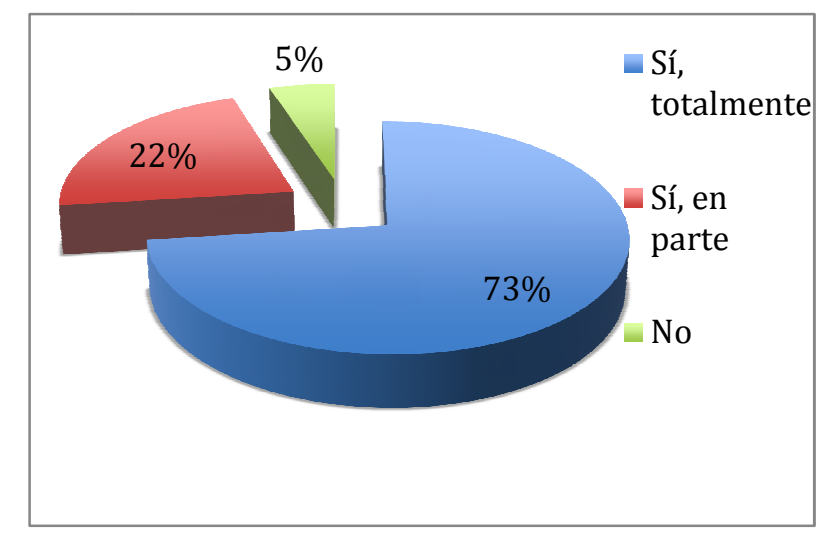

Fuente: Elaboración propia.

Por otro lado, la existencia de un buen clima laboral es fundamental en todas las organizaciones a la hora de garantizar un buen funcionamiento y unos estándares de productividad y calidad adecuados. Por ello, la relación que mantienen los miembros de una organización, como las sociedades laborales, de tamaño reducido y donde el trato con los compañeros es constante, resulta fundamental para la consecución del éxito empresarial. A este respecto el 90,73\% de los socios manifiesta que entre los miembros de la organización existe una relación de amistad, frente a un 9,27\% de los casos donde afirman que la relación es únicamente profesional. 
Gráfico 8. Tipo de relación entre los socios y compañeros

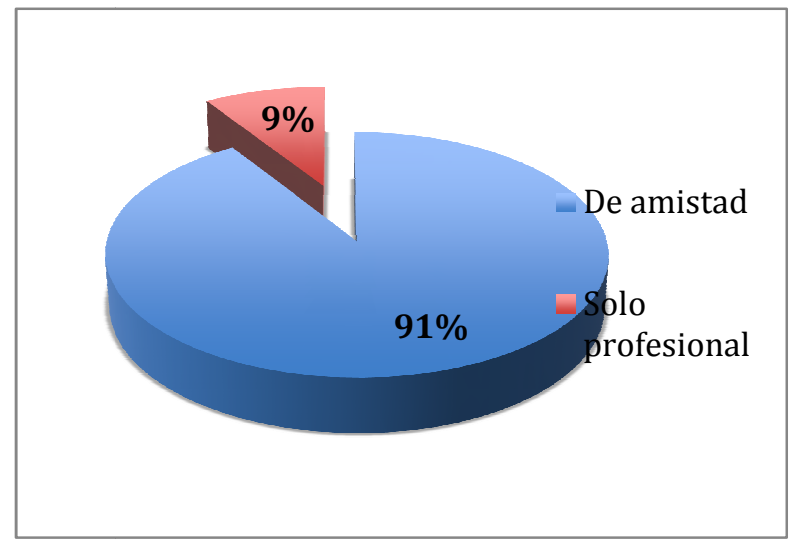

Fuente: Elaboración propia.

En cuanto al proceso de toma de decisiones, el 78,37\% de las sociedades laborales manifiestan que la toma de decisiones se lleva a cabo entre todos los socios, mientras que el $21,63 \%$ de las mismas afirma que en su caso la toma de decisiones la realiza cada socio dentro de su área de responsabilidad.

Gráfico 9. Flujos de comunicación interna

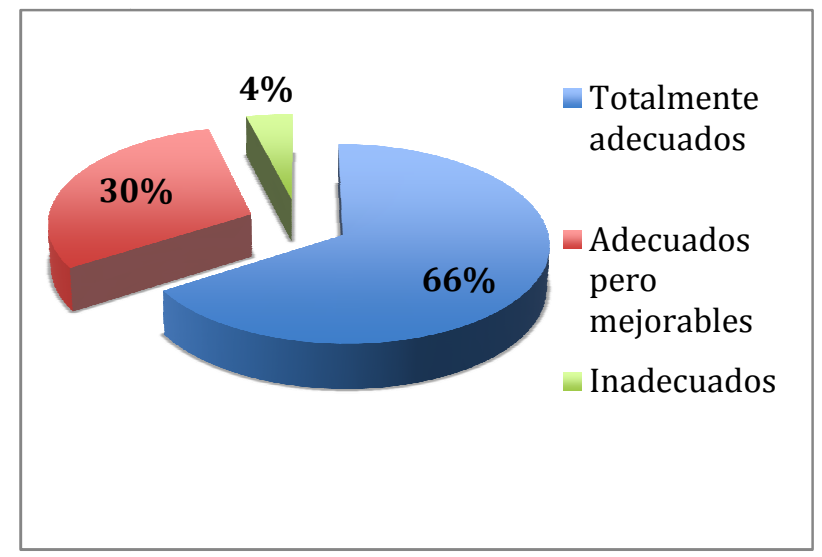

Fuente: Elaboración propia.

Otra variable trascendente a considerar es la que constituyen los procesos de comunicación interna. A este respecto, el 66,01\% de las sociedades laborales considera que su comunicación interna actual es correcta y, por tanto, adecuada. Por otro lado, un 30,06\% de las mismas afirma que su comunicación es adecuada pero mejorable, mientras que únicamente un 3,93\% de las sociedades ha manifestado que sus procesos de comunicación interna son inadecuados. Estos datos manifiestan la facilidad que encuentran las pequeñas 
empresas para simplificar sus procesos de comunicación interna haciéndolos más eficaces y ágiles, lo que supone una importante ventaja en el desarrollo de su actividad, evitar malentendidos y favorecer su supervivencia.

Gráfico 10. Proceso de toma de decisiones

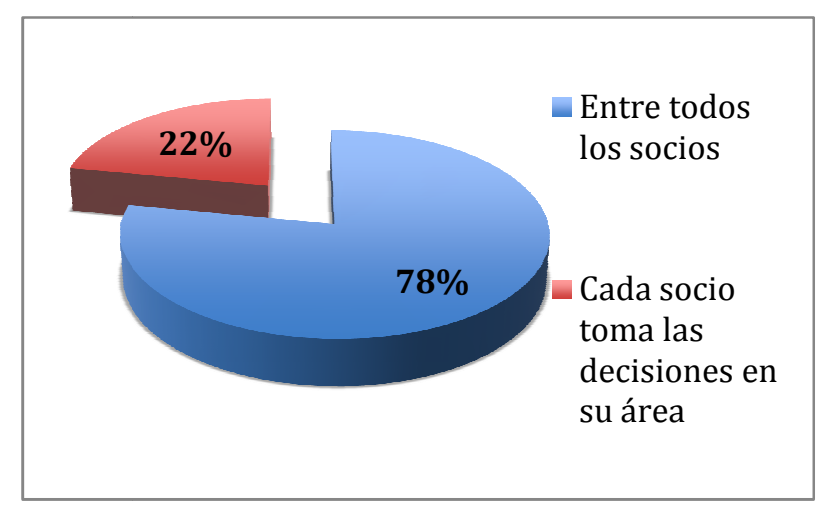

Fuente: Elaboración propia

Por último, para las sociedades laborales la innovación no sólo es vista como un aspecto de gran importancia sino que, además, supone para ellas un reto a la hora de posicionarse de forma competitiva en el mercado globalizado.

Gráfico 11. La innovación como ventaja competitiva

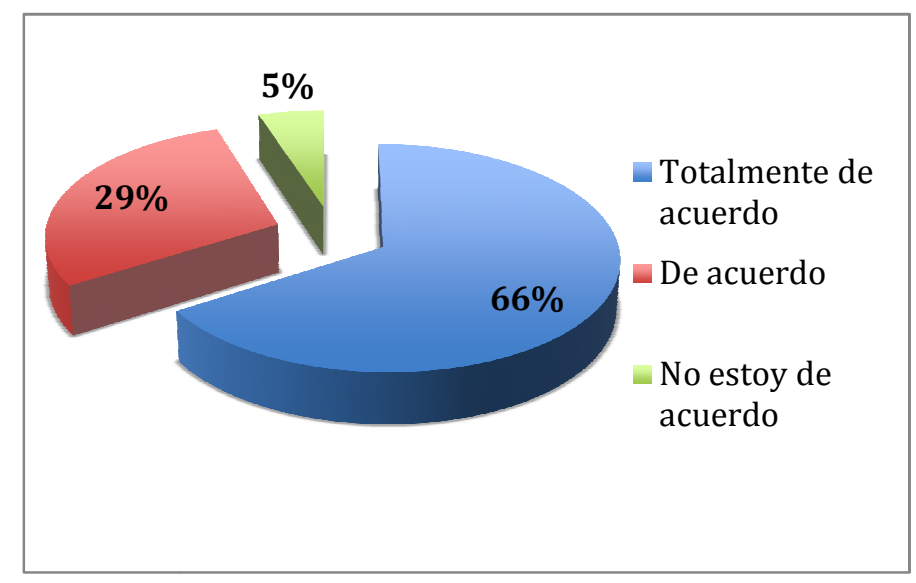

Fuente: Elaboración propia.

Así, entendiendo la Innovación como cualquier proceso diferenciador del producto, de los procesos de producción, etc. y no únicamente como el I+D+I, la mayoría de las sociedades laborales $(66,29 \%)$ está totalmente de acuerdo en que ésta representa una posición estratégica para la consecución de los objetivos de la empresa y su afianzamiento en el mercado, mostrándose, por tanto, como una importante ventaja competitiva a considerar. Por su parte, 
un $28,37 \%$ considera que la innovación es muy importante pero cree que su influencia por sí sola no constituye la diferenciación necesaria de la empresa en la búsqueda de la ventaja competitiva. Por el contrario, solo un $5,34 \%$ de las sociedades laborales no considera a la innovación como fuente de ventaja competitiva.

\section{CONCLUSIÓN: PERFIL GENERAL DEL EMPRENDEDOR DE SOCIEDADES LABORALES EN ANDALUCÍA}

De todo lo anterior se puede establecer que el perfil tipo del emprendedor de Sociedades Laborales en Andalucía se identifica con un varón de 35 años, socio trabajador y de nacionalidad española, con residencia en la provincia de Sevilla, estudios de Enseñanza Secundaria (ESO) o equivalente, que decide emprender una actividad empresarial preferencialmente en el sector terciario o de servicios.

Por otra parte, se pueden añadir, además, los siguientes rasgos característicos:

- La mayoría decide emprender por la satisfacción personal que supone crear su propio medio de vida (motivación positiva).

- Se consideran responsables, perseverantes, optimistas y con un alto grado de autoconfianza.

- Están convencidos de que cuentan con la formación profesional, experiencia y conocimientos idóneos para el desarrollo de sus actividades..

- Planifican de manera sistemática y definen con claridad las funciones y responsabilidades de cada socio.

- Entre ellos existe una relación que se puede considerar como de amistad, además de la profesional.

- La toma de decisiones se desarrolla, normalmente, de manera conjunta entre todos los socios, para lo cual existe una comunicación interna adecuada.

- Por último, consideran la innovación como un aspecto muy importante para el desarrollo de sus actividades y para su posicionamiento competitivo en el mercado. 


\section{BIBLIOGRAFÍA}

AGUIRRE SÁDABA, A. y SANTOS ARREBOLA, J. L. (2004) Orientación al Mercado. Guía para la creación de empresas en Andalucía. Málaga: Bic Euronova.

ALGUACIL MARÍ, M. P. (2007) La tributación de las Sociedades Laborales. Madrid: Editorial Aranzadi. Colección: Derecho Fiscal. Monografías Aranzadi no 478.

AMABILE, T. M. (1997) Entrepreneurial Creativity. Through motivational synergy. Journal of Creativity Behavior, $\mathrm{n}^{\circ} 31$, pp. 18-25.

BARBA SÁNCHEZ, V. y MARTÍNEZ RUIZ, M. P. (2006) Cambios en el modelo de desarrollo económico y creación de empresas. El emprendedor como factor clave del proceso de cambio. Boletín Económico de ICE, nº 2682, pp. 15-28.

BLÁZQUEZ SANTANA, F.; DORTA VELÁZQUEZ, J. A.; y VERONA MARTEL, M. C. (2006) Factores del crecimiento empresarial. Especial referencia a las pequeñas y medianas empresas. INNOVAR. Revista de Ciencias Administrativas y Sociales., Vol. 16, n 26, pp. 42-56.

BURCH, J. G. (1986) Entrepreneurship. New York: Ed. John Wiley and Sons.

CASTILlO CLAVERO, A. M., ET. AL. (2003) Introducción a la economía y administración de empresas. Madrid: Pirámide

CASTILLO, V.; RIVAS, D.; ROJO, S.; y ROTONDO, S. (2006) La creación de nuevas empresas durante la etapa postconvertibilidad (2003-2005): impacto sobre el empleo asalariado registrado. Dirección General de Estudios y Estadísticas Laborales. Subsecretaría de Programación Técnica y Estudios Laborales. Ministerio de Trabajo, Empleo y Seguridad Social. http://www.aaep.org.ar/anales/buscador.php

CANO LÓPEZ, A. (2002) Teoría jurídica de la economía social: la Sociedad Laboral: una forma jurídica de empresa de economía social. Madrid: CES

CAPRARA, G. V.; BARBARANELLI, C. y BORGOGNI, L. (1998) BFQ. Cuestionario Big Five. Madrid: TEA

CEPES (1993) Manifiesto-Programa de la Economía Social. Madrid: I Congreso de la Economía Social. Comité Español Permanente de la Economía Social.

CIRUELA, A. M. (2006) La función directiva en las cooperativas agrarias. Estudio empírico 
en las sociedades cooperativas cafetaleras de Costa Rica. CIRIEC-España, Revista de Economía Pública, Social y Cooperativa, nº 55, agosto, pp. 253-288.

CODURAS MARTÍNEZ, A. (2006) La motivación para emprender en España. Ekonomiaz, no 62, $2^{\circ}$ Cuatrimestre, pp. 12-39.

COLL SERRANO, V. y CUÑAT GIMÉNEZ, R. (2006) Análisis de los factores que influyen en el proceso de creación de una cooperativa de trabajo asociado. REVESCO. Revista de Estudios Cooperativos, Primer cuatrimestre, $\mathrm{n}^{\circ}$ 88, pp. 128-161.

CONGREGADO, E.; HERNÁNDEZ, L.; MILLÁN, J. M.; RAYMOND, J. L.; ROIG, J. L.; SALAS, V.; SÁNCHEZ-ASÍN, J. L. y SERRANO, L. (2008) El capital humano y los emprendedores en España. Cuaderno $\mathrm{n}^{\circ}$ 93: El perfil del emprendedor en España, Septiembre, Valencia: Bancaja, Valencia.

CONSEJO ECONÓMICO Y SOCIAL (2005) El proceso de creación de empresas y el dinamismo empresarial. Informe 5/2005, CES.

DÍEZ DE CASTRO, E., GALÁN GONZÁLEZ, J. L., LANDA BERCEBAL, F. J., LEAL MILLÁN, A. (1995) La empresa en Andalucía. Madrid: Cívitas

GARCÍA SÁNCHEZ, A. y WANDOSELL, G. (2004) Perfil del emprendedor y proceso de creación de empresas en la Región de Murcia. Murcia: Instituto de Fomento de la Región de Murcia.

GARCÍA TABUENCA, A.; CRESPO ESPERT, J. L.; MARTÍ, F. P. y CRECENTE ROMERO, F. (2007) Perfil de los empresarios y resultados de sus empresas. Madrid: Serie Documentos de Trabajo. 05/2007, Instituto Universitario de Análisis Económico y Social, Universidad de Alcalá, pp. 1- 30.

GONZÁLEZ DOMÍNGUEZ, F. J. (2006) Creación de empresas. Guía del emprendedor. Madrid: Pirámide.

HISRICH, R. D.; PETERS, M. P. y SHEPHERD, D. A. (2005) Entrepreneurship. Emprendedores. Madrid: Mc Graw Hill.

LEJARRIAGA PÉREZ DE LAS VACAS, G. (2002) Las empresas de participación como base del contenido de la economía social en el marco de la Unión Europea. En VÁRGAS, A. y LEJARRIAGA, G. (Coord.) Las empresas de participación en Europa: el reto del 
siglo XXI (En homenaje al Prof. Dr. Carlos GARCÍA-GUTIÉRREZ FERNÁNDEZ). Madrid: Escuela de Estudios Cooperativos, pp. 83-99.

LEY 44/2015, DE 14 DE OCTUBRE, DE SOCIEDADES LABORALES Y PARTICIPADAS. Boletín Oficial del Estado (BOE), n 247, de 15 de octubre de 2015, pp. $95747-95763$.

LEY 5/2011, DE 29 DE MARZO, DE ECONOMÍA SOCIAL. Boletín Oficial del Estado (BOE) n n $^{\circ}$, de 30 de marzo de 2011, pp. 33023-33033.

LÓPEZ GANDÍA, JUAN (2006) Las sociedades laborales y la aplicación del derecho del trabajo. Valencia: Tirant lo Blanch Colección Laboral,.

MARTÍN LÓPEZ, S.; LEJARRIAGA PÉREZ DE LAS VACAS, G. \& ITURRIOZ DEL CAMPO, J.. (2005a) Valoración financiera de las propuestas alternativas de modificación del régimen de capitalización por desempleo para el colectivo de los trabajadores autónomos. REVESCO. Revista de Estudios Cooperativos, nº 87, pp. 139-164.

MARTÍN LÓPEZ, S.; LEJARRIAGA PÉREZ DE LAS VACAS, G. \& ITURRIOZ DEL CAMPO, J.. (2005b) Casuística de acceso a la actualización de la prestación por desempleo: bases para un modelo de decisión. CIRIEC-España, Revista de economía pública, social y cooperativa, (52), 307-333.

MARTÍN LÓPEZ, S.; LEJARRIAGA PÉREZ DE LAS VACAS, G. \& ITURRIOZ DEL CAMPO, J.. (2007) La naturaleza del capital social como aspecto diferenciador entre las sociedades cooperativas y las sociedades laborales. CIRIEC-España, Revista de economía pública, social y cooperativa, (58), pp. 59-82.

MARTÍN LÓPEZ, S.; LEJARRIAGA PÉREZ DE LAS VACAS, G. (2011) Las empresas de participación de trabajo asociado ante los desafíos del futuro en un contexto de crisis económica: propuestas de actuación. CIRIEC-España, Revista de Economía Pública, Social y Cooperativa, $\mathrm{n}^{\mathrm{o}}$ 72, Octubre 2011, pp. 239-261.

MARTÍN CRUZ, N.; HERNANGÓMEZ BARAHONA, J. y RODRÍGUEZ ESCUDERO, A. I. (2005) Análisis de la formación y la experiencia laboral como determinantes del espíritu emprendedor de los estudiantes universitarios. Revista Asturiana de Economía, RAE, $\mathrm{n}^{\circ}$ 34, pp. 131-145. 
MELGAREJO, Z.; ARCELUS, F.; SIMON, K. (2007) Desempeño económico: diferencias de pequeñas empresas clasificadas según la estructura de la propiedad del capital. REVESCO. Revista de Estudios Cooperativos, $\mathrm{n}^{\circ}$ 93, tercer cuatrimestre 2007, pp. 7-38.

MORIANO LEÓN, J. A. (2005) El perfil psicosocial del emprendedor: un estudio realizado en Castilla y León. Madrid: CES, Consejo Económico y Social, Colección de Estudios

MORRIS, M. y KURATKO, D. (2002) Corporate entrepreneurship entrepreurial development within organiztions. San Diego: Harcourt College Publishers.

OLAMENDI, G. (1998) Cómo crear una empresa y triunfar en el intento. Bilbao: Olamendi Ediciones.

PEÑA LEGAZKUE, I. (2008) El efecto de factores externos e internos de la empresa en la supervivencia de las PYME españolas. En RODRÍGUEZ CASTELLANOS, A. (dir.) et al. Creación, supervivencia, crecimiento e internacionalización de las PYME jóvenes en España: 1995-2006. Madrid: Dirección General de Política de la PYME.

PÉREZ FERNÁNDEZ, E. (2002) La participación como elemento constitutivo de las empresas de la Nueva Economía Social. CIRIEC-España, Revista de Economía Pública, Social y Cooperativa, $\mathrm{n}^{\circ} 40$, abril 2002, pp. 141-163.

PINILLOS COSTA, M. J. (2007) Un estudio de la influencia de clientes y financiadores en el crecimiento de las empresas. El comportamiento de la empresa ante entornos dinámicos. XIX Congreso anual y XV Congreso Hispano Francés de AEDEM, vol. 1, 2007 (Ponencias), pág. 45.

PlAZA ANGUlO, J. J.; y ÁlVAREZ CORTÉS, J. C. (2008) Prestaciones de seguridad social. El desempleo en su modalidad de pago único como ayuda a nuevos emprendedores. Temas Laborales. Revista Andaluza de Trabajo y Bienestar social, $\mathrm{n}^{\circ}$ 95, pp. 283-29.

SEGARRA BLASCO, A.; y TERUEL CARRIZOSA, M. (2007) Creación y supervivencia de las nuevas empresas en las manufacturas y los servicios. Economía Industrial, no 363 , 2007, pp. 47-58.

SHAPERO, A. y SOKOL, L. (1982) The social dimensions of entrepreneurship. En Kent, C.A., Sexton, D.L. y Vesper, K. Ed. Encyclopedia of Entrepreneurship, Ed. Prentice Hall, pp.72-89.

REVESCO No 122 - Tercer Cuatrimestre 2016 - ISSN: 1885-8031 - www.ucm.es/info/revesco 
SUÁREZ SUÁREZ, A. S. (1992) Diccionario de Economía y Administración. Madrid: Mc Graw-Hill.

STEVENSON, H. H.; ROBERTS, M. y GROUSBECK, H. I. (1985) New Business Ventures and the Entrepreneur. New York: Ed. R. D. Irwin.

VALPUESTA GASTAMINZA, E. M. y BARBERENA BELZUNCE, I. (1998) Las Sociedades Laborales: aspectos societarios, laborales y fiscales. Pamplona: Editorial Aranzadi.

VANDERWERF, P. y BRUSH, C. (1989) Toward agreement on the focus of entrepreneurship research: progress without definition. Proceedings of The National Academy of Management, Conference, Washington D. C., USA.

VECIANA VERGES, J. M. (2005) La creación de empresas. Un enfoque gerencial. Barcelona: Colección Estudios Económicos, n³ 33, La Caixa.

VILLALBA CABELlO, F. (dir.); MUÑOZ LÓPEZ, J. A. (coord.); MORILLA GARCÍA, F.; NIETO LOBO, E.; PÉREZ GUIRADO, J. A.; y ROMÁN JOBACHO, M. L. (2007) Análisis económico-financiero de la empresa andaluza. Sevilla: Instituto de Análisis Económico y Empresarial de Andalucía.

WENNEKERS, S. y THURIK, R. (1999) Linking Entrepreneurship and Economic Growth. Small Business Economics, Vol. 13, nº 1, August, pp. 27-56. 